\title{
HOUSEHOLD WATER CONSUMPTION: SUMMARY OF EMPIRICAL RESULTS
}

In the following Tables, the empirical results reviewed for this report are related to the household water consumption sector are summarised. Table C.1 provides a brief overview of findings related to the effectiveness of various policy instruments. Table C.2 summarises findings related to the effects of other variables, especially demographic characteristics: if the effect of a variable is significant, the direction of the effect (positive or negative) is indicated in brackets (if the effect is insignificant, the direction of the effect is not given). Table C.3 includes the various "own-price" and income elasticities of demand for water. 
TABLE C.1: Overview of pricing and non-pricing policies for residential water demand

\begin{tabular}{|c|c|c|c|}
\hline Authors (year) & Dependent variables & Independent policy variables & Data and country \\
\hline $\begin{array}{l}\text { Billings and Agthe } \\
(1980)\end{array}$ & $\begin{array}{l}\text { monthly water consumption per } \\
\text { household }\end{array}$ & - $\quad$ increasing block rates & $\begin{array}{l}\text { Tucson, Arizona, USA } \\
\text { (Jan } 1974 \text { to Sept 1977) }\end{array}$ \\
\hline $\begin{array}{l}\text { Chicoine and } \\
\text { Ramamurthy (1986) }\end{array}$ & $\begin{array}{l}\text { quantity of water purchased per } \\
\text { month }\end{array}$ & - declining block-price schedule & $\begin{array}{l}59 \text { rural water districts, } \\
\text { Illinois, USA }\end{array}$ \\
\hline Cummings et al. (2005) & $\begin{array}{l}\text { marginal quantity (amount of } \\
\text { water used beyond the level of last } \\
\text { consumed block) }\end{array}$ & - $\quad$ block-rate pricing & $\begin{array}{l}\text { aggregate data, } \\
28 \text { coastal counties, Georgia } \\
\text { (2003 to 2005) }\end{array}$ \\
\hline Dandy et al. (1997) & annual water consumption & $\begin{array}{l}\text { - marginal price (below free allowance level, marginal price } \\
\text { is zero, and above allowance level, it is the unit price of } \\
\text { water) } \\
\text { dummy for reduction in free allowance level of water } \\
\text { (before 1991-92, annual allocation of water calculated as } \\
\text { percentage of property value; during 1991-92, a constant } \\
\text { quantity of } 136 \mathrm{kl} \text { of water allocated to households, which } \\
\text { is much less than before for average consumer) }\end{array}$ & $\begin{array}{l}\text { metropolitan area of Adelaide, } \\
\text { Australia } \\
\text { (1978 to 1992) }\end{array}$ \\
\hline $\begin{array}{l}\text { Domene and Sauri } \\
\text { (2005) }\end{array}$ & $\begin{array}{l}\text { Per-capita water consumption per } \\
\text { day }\end{array}$ & - $\quad$ heterogeneous pricing schemes & $\begin{array}{llr}\text { cross-sectional } & \text { data } & \text { from } \\
\text { metropolitan } & \text { region } & \text { of } \\
\text { Barcelona (2003) } & & \end{array}$ \\
\hline Espiñeira (2000) & $\begin{array}{l}\text { monthly average water use per } \\
\text { account }\end{array}$ & $\begin{array}{l}\text { - increasing block rate (a fixed quota with a minimum free } \\
\text { allowance plus a second block charged at a single rate or } \\
\text { several blocks charged at increasing rates) }\end{array}$ & $\begin{array}{l}\text { monthly panel data, } \\
\text { north-west Spain }\end{array}$ \\
\hline $\begin{array}{l}\text { Espiñeira and Nauges } \\
\text { (2004) }\end{array}$ & $\begin{array}{lc}\text { Per-capita } \quad \text { average } & \text { domestic } \\
\text { consumption per month } & \end{array}$ & $\begin{array}{ll}\text { - } & \text { increasing block rate } \\
\text { - } & \text { restricted daily water supply hours during severe drought } \\
\text { periods } \\
\text { - } & \text { temporary outdoor use bans }\end{array}$ & $\begin{array}{l}\text { Seville, Spain } \\
\text { (1991 to 1999) }\end{array}$ \\
\hline
\end{tabular}




\begin{tabular}{|c|c|c|c|}
\hline Authors (year) & Dependent variables & Independent policy variables & Data and country \\
\hline Gaudin (2006) & $\begin{array}{lll}\text { Per-capita } & \text { annual } & \text { water } \\
\text { consumption } & & \end{array}$ & $\begin{array}{l}\text { - } \quad \text { information dummy for marginal price info (unit price of } \\
\text { water is given next to units consumed) } \\
\text { - } \quad \text { information dummy for price info (full price information of } \\
\text { - } \quad \text { information is given anywhere in the bill) } \\
\text { - } \quad \text { information dummy for quantity consumed info } \\
\text { - } \quad \text { informerage, gas or electricity, and high-frequency billing } \\
\text { - infion dummy for increased block rate }\end{array}$ & cross-sectional data, USA \\
\hline Krause et al. (2003) & $\begin{array}{l}\text { percentage of water resource stock } \\
\text { drawn }\end{array}$ & $\begin{array}{l}\text { - allowing consumption from a finite stock of water, in } \\
\text { presence of explicit scarcity (it shows that consumption of } \\
\text { water depends on type of consumer) }\end{array}$ & $\begin{array}{l}\text { classroom experiment } 0114 \\
\text { people consisting of three } \\
\text { groups: students, working } \\
\text { adults, and retired people }\end{array}$ \\
\hline $\begin{array}{l}\text { Mazzanti and Montini } \\
\text { (2006) }\end{array}$ & Per-capita water consumption & $\begin{array}{l}\text { - increasing block-rate structure (central price is used in the } \\
\text { equation) }\end{array}$ & $\begin{array}{l}\text { Emilia-Romagna, Italy } \\
\text { (1998 to 2001) }\end{array}$ \\
\hline $\begin{array}{l}\text { Nauges and Thomas } \\
(2000)\end{array}$ & $\begin{array}{l}\text { water consumption in local } \\
\text { community }\end{array}$ & $\begin{array}{ll} & \text { two-part tariff } \\
\text { - } & \text { existence of own water meter in single-unit houses }\end{array}$ & $\begin{array}{l}\text { panel data from } 116 \\
\text { communities, eastern France } \\
\text { (1988 to 1993) }\end{array}$ \\
\hline $\begin{array}{l}\text { Nauges and Thomas } \\
\text { (2003) }\end{array}$ & $\begin{array}{l}\text { annual consumption of water } \\
\text { (intertemporal choice) }\end{array}$ & $\begin{array}{ll}\text { - } & \text { two-part tariff (a fixed charge corresponding to connection } \\
& \text { fee and a price per unit of water consumed) } \\
\text { - } & \text { lagged consumption }\end{array}$ & $\begin{array}{l}\text { panel data of } 116 \text { communities, } \\
\text { eastern France } \\
\text { (1988 to 1993) }\end{array}$ \\
\hline $\begin{array}{ll}\text { Nieswiadomy } & \text { and } \\
\text { Molina (1989) } & \end{array}$ & monthly water consumption & $\begin{array}{ll}- & \text { increasing block rate (1976 to } 1980 \text { ) } \\
\text { - } & \text { decreasing block rate (1981 to } 1985 \text { ) }\end{array}$ & $\begin{array}{l}\text { monthly data, } \\
\text { City of Denton, Texas } \\
\text { (1976 to 1985) }\end{array}$ \\
\hline Pint (1999) & bi-monthly water use & - $\quad$ increasing block rate & $\begin{array}{l}\text { Alameda County Water District, } \\
\text { California } \\
\text { (Jan } 1982 \text { to Jul 1992) }\end{array}$ \\
\hline
\end{tabular}




\begin{tabular}{|c|c|c|c|}
\hline Authors (year) & Dependent variables & Independent policy variables & Data and country \\
\hline $\begin{array}{l}\text { Renwick and Archibald } \\
\text { (1998) }\end{array}$ & water consumption per household & 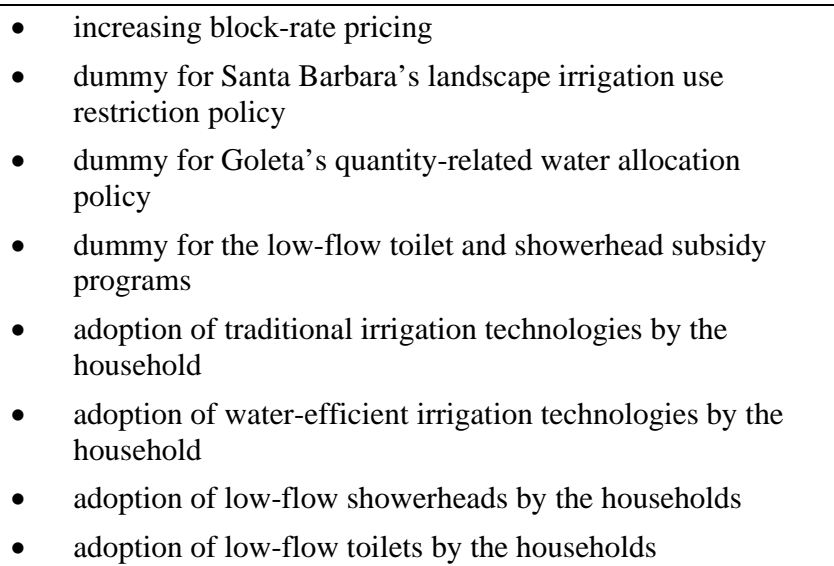 & $\begin{array}{l}\text { household survey data, } \\
\text { Santa Barbara and Goleta, } \\
\text { California } \\
\text { (1985 to 1992) }\end{array}$ \\
\hline Renwick et al. (1998) & $\begin{array}{l}\text { average household monthly water } \\
\text { use }\end{array}$ & 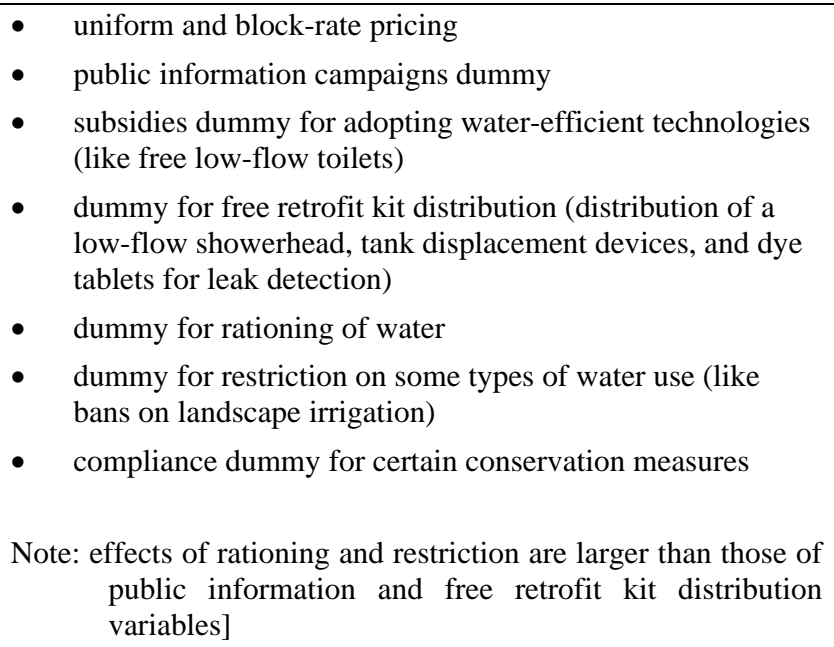 & $\begin{array}{l}\text { eight urban water agencies, } \\
\text { California, USA } \\
\text { (1989 to 1996) }\end{array}$ \\
\hline $\begin{array}{l}\text { Schefter and David } \\
\text { (1985) }\end{array}$ & $\begin{array}{l}\text { total quantity of water delivered to } \\
\text { the residential users }\end{array}$ & - $\quad$ various price schedules & $\begin{array}{l}\text { aggregate data, } \\
131 \text { Wisconsin communities }\end{array}$ \\
\hline
\end{tabular}




\begin{tabular}{|c|c|c|c|}
\hline Authors (year) & Dependent variables & Independent policy variables & Data and country \\
\hline $\begin{array}{l}\text { Strand and Walker } \\
(2005)\end{array}$ & average water consumption & $\begin{array}{ll}\text { - } & \text { block-rate pricing } \\
\text { - } & \text { rationing of water through reduced service hours }\end{array}$ & $\begin{array}{l}\text { survey data, } \\
17 \text { Central American cities }\end{array}$ \\
\hline Taylor et al. (2004) & $\begin{array}{l}\text { quantity of domestic water } \\
\text { demanded }\end{array}$ & $\begin{array}{l}\text { - various price schedules (flat-rate pricing, increasing block, } \\
\text { decreasing block, and unmetered fixed fees) } \\
\text { - } \quad \text { water conservation programs }\end{array}$ & $\begin{array}{l}\text { cross-sectional data } \\
34 \text { utilities from Colorado water } \\
\text { districts }\end{array}$ \\
\hline $\begin{array}{l}\text { Whittington et al. } \\
\text { (1990) }\end{array}$ & $\begin{array}{l}\text { probability that a household's } \\
\text { WTP for a public stand-post } \\
\text { (private connection) falls within a } \\
\text { specific interval }\end{array}$ & $\begin{array}{l}\text { installation of private connections when public water } \\
\text { supply posts are in place }\end{array}$ & $\begin{array}{l}\text { Laurent, southern Haiti } \\
\text { (1986) }\end{array}$ \\
\hline Wong (1972) & average per-capita water demand & $\begin{array}{ll}\text { - } & \text { flat rate of water per } 1000 \text { gallons (Chicago) } \\
\text { - } & \text { block rates (suburban communities) }\end{array}$ & $\begin{array}{l}\text { Time-series analysis, Chicago } \\
\text { and nearby communities, Illinois } \\
\text { (1951 to 1961) } \\
\text { cross-sectional analysis with } \\
\text { four community sizes }\end{array}$ \\
\hline Woo (1994) & monthly per-capita water use & $\begin{array}{ll}\text { - } & \text { service interruption of water (to save water reserves) } \\
\text { - } & \text { inverted block-rate pricing }\end{array}$ & $\begin{array}{l}\text { Hong Kong } \\
\text { (1973 to 1990) }\end{array}$ \\
\hline
\end{tabular}


TABLE C.2: Econometric studies on the determinants of residential water use

\begin{tabular}{|c|c|c|c|}
\hline Authors (year) & Dependent variables & $\begin{array}{c}\text { Independent variables other than policy variables (mostly demographic } \\
\text { characteristics) }\end{array}$ & Data and Country \\
\hline $\begin{array}{l}\text { Billings and Agthe } \\
(1980)\end{array}$ & $\begin{array}{l}\text { monthly water consumption } \\
\text { per household }\end{array}$ & $\begin{array}{ll}\text { - } & \text { marginal price }(-) \\
\text { - } & \text { difference variable }(-) \\
\text { - } & \text { personal income }(+) \\
\text { - } & \text { weather variable (evapotranspiration - rainfall) }(+)\end{array}$ & $\begin{array}{l}\text { Tucson, Arizona, USA } \\
\text { (Jan } 1974 \text { to Sept 1977) }\end{array}$ \\
\hline $\begin{array}{l}\text { Chicoine and } \\
\text { Ramamurthy (1986) }\end{array}$ & $\begin{array}{l}\text { quantity of water purchased per } \\
\text { month }\end{array}$ & $\begin{array}{ll}\text { - } & \text { income (monthly income of the household - difference variable) }(+) \\
\text { - } & \text { marginal price }(-) \\
\text { - } & \text { decomposed price }(-) \\
\text { - } & \text { number of persons in a household }(+) \\
\text { - } & \text { number of bathrooms }(+)\end{array}$ & $\begin{array}{l}59 \text { rural water districts, } \\
\text { Illinois, USA }\end{array}$ \\
\hline $\begin{array}{l}\text { Cummings et al. } \\
\text { (2005) }\end{array}$ & $\begin{array}{l}\text { marginal quantity (amount of } \\
\text { water used beyond the level of } \\
\text { last consumed block) }\end{array}$ & 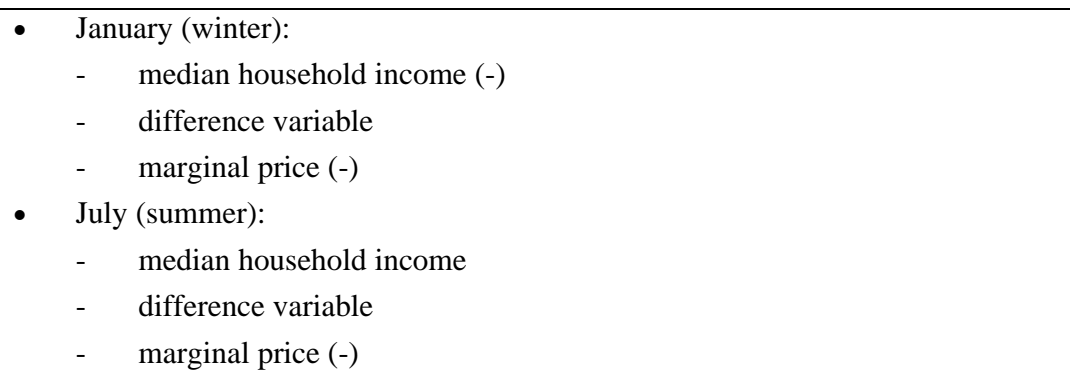 & $\begin{array}{l}\text { aggregate data, } \\
28 \text { coastal counties, Georgia } \\
\text { (2003 to 2005) }\end{array}$ \\
\hline
\end{tabular}




\begin{tabular}{|c|c|c|c|}
\hline Authors (year) & Dependent variables & $\begin{array}{c}\text { Independent variables other than policy variables (mostly demographic } \\
\text { characteristics) }\end{array}$ & Data and Country \\
\hline Dandy et al. (1997) & annual water consumption & $\begin{array}{ll}\text { - } & \text { marginal price }(-) \\
\text { - } & \text { difference variable }(-) \\
\text { - } & \text { capital value of property (proxy for income) }(+) \\
\text { - } & \text { plot size } \\
\text { - } & \text { number of residents per household }(+) \\
\text { - } & \text { number of rooms in the house }(+) \\
\text { - } & \text { swimming pool ownership }(+) \\
\text { - } & \text { moisture deficit (= potential evapotranspiration }-0.6 \text { times rainfall) }(+) \\
\text { - } & \text { lagged consumption (annual consumption in previous year) }(+) \\
\text { - } & \text { dummy for change in free allowance level (higher free allowance level prior } \\
& \text { to } 1992 \text { than in 1992) }(-)\end{array}$ & $\begin{array}{l}\text { metropolitan area of Adelaide, } \\
\text { Australia } \\
\text { (1978 to 1992) }\end{array}$ \\
\hline $\begin{array}{l}\text { Domene and Sauri } \\
\text { (2005) }\end{array}$ & $\begin{array}{l}\text { Per-capita water consumption } \\
\text { per day }\end{array}$ & $\begin{array}{ll}\text { - } & \text { average water price } \\
\text { - } & \text { income of the family unit }(+) \\
\text { - } & \text { housing type dummy (high-density; mid-density; low-density) }(+) \\
\text { - } & \text { number of members per household }(-) \\
\text { - } & \text { house size } \\
\text { - } & \text { garden size } \\
\text { - } & \text { garden necessity of water }(+) \\
\text { - } & \text { swimming pool dummy }(+) \\
\text { - } & \text { consumer behaviour index }(-)\end{array}$ & $\begin{array}{l}\text { cross-sectional data } \\
\text { metropolitan region of Barcelona } \\
\text { (2003) }\end{array}$ \\
\hline
\end{tabular}




\begin{tabular}{|c|c|c|c|}
\hline Authors (year) & Dependent variables & $\begin{array}{l}\text { Independent variables other than policy variables (mostly demographic } \\
\text { characteristics) }\end{array}$ & Data and Country \\
\hline Espiñeira (2000) & $\begin{array}{l}\text { monthly average water use per } \\
\text { account }\end{array}$ & $\begin{array}{ll}\text { - } & \text { marginal price }(-) \\
\text { - } & \text { difference variable }(-) \\
\text { - } & \text { number of rainy days in the month }(-) \\
\text { - } & \text { average temperature in each month }(+) \\
\text { - } & \text { number of billing periods in the year } \\
\text { - } & \text { average number of members of the households } \\
\text { - } & \text { family disposable income } \\
\text { - } & \text { number of units charged regardless of actual use (takes into account impact of } \\
& \text { free allowance level) }(+) \\
\text { - } & \text { percentage of population over age } 64 \\
\text { - } & \text { percentage of dwellings statistically regarded as main residence }\end{array}$ & $\begin{array}{l}\text { monthly panel data, } \\
\text { north-west Spain }\end{array}$ \\
\hline $\begin{array}{l}\text { Espiñeira and Nauges } \\
\text { (2004) }\end{array}$ & $\begin{array}{l}\text { Per-capita average domestic } \\
\text { consumption per month }\end{array}$ & $\begin{array}{ll}\text { - } & \text { minimum threshold level of water }(+) \\
\text { - } & \text { income/price ratio (insignificant with variable threshold level assumption) } \\
\text { - } & \text { outdoor usage bans } \\
\text { - } & \text { average past consumption (with variable threshold level assumption) }(+) \\
\text { - } & \text { supply restrictions }(-) \\
\text { - } & \text { normal value of rainfall }(-)\end{array}$ & $\begin{array}{l}\text { Seville, Spain } \\
\text { (1991 to 1999) }\end{array}$ \\
\hline
\end{tabular}




\begin{tabular}{|c|c|c|c|}
\hline Authors (year) & Dependent variables & $\begin{array}{l}\text { Independent variables other than policy variables (mostly demographic } \\
\text { characteristics) }\end{array}$ & Data and Country \\
\hline Gaudin (2006) & $\begin{array}{l}\text { per-capita annual water } \\
\text { consumption }\end{array}$ & $\begin{array}{ll}\text { - } & \text { average price }(-) \\
\text { - } & \text { income }(+) \\
\text { - } & \text { average household size }(+) \\
\text { - } & \text { density }(-) \\
\text { - } & \text { average annual precipitation }(-) \\
\text { - } & \text { number of days when temperature is above } 90^{0} \mathrm{~F}(+) \\
\text { - } & \text { information dummy for marginal price info (unit price of water is given next } \\
\text { - } & \text { to the units consumed) }(-) \\
\text { - } & \text { information dummy for price info (full price information of water is given } \\
\text { - } & \text { information dummy for quantity consumed info } \\
\text { - } & \text { information dummy for combined water billing with sewerage, gas or } \\
\text { information dummy for the increased block rate }\end{array}$ & $\begin{array}{l}\text { cross-sectional community level } \\
\text { data, USA } \\
\text { (1995 to 1996) }\end{array}$ \\
\hline Krause et al. (2003) & $\begin{array}{l}\text { percentage of water resource } \\
\text { stock drawn }\end{array}$ & $\begin{array}{ll}\text { - } & \text { price }(-) \\
\text { - } & \text { age (in case of retirees only) }(-) \\
\text { - } & \text { female } \\
\text { - } & \text { Hispanic (among retirees, Hispanics use more water than their Caucasian } \\
& \text { counterparts) }(+) \\
\text { - } & \text { political or religious affiliation } \\
\text { - } & \text { attendance in religious services (retirees consume more water) }(+) \\
\text { - } & \text { years of schooling } \\
\text { - } & \text { risk preferences (risk-averse working adults and risk-loving retirees use less } \\
& \text { water) }(-)\end{array}$ & $\begin{array}{l}\text { classroom experiment of } 114 \\
\text { people consisting of three groups: } \\
\text { students, working adults and } \\
\text { retired people }\end{array}$ \\
\hline
\end{tabular}




\begin{tabular}{|c|c|c|c|}
\hline Authors (year) & Dependent variables & $\begin{array}{l}\text { Independent variables other than policy variables (mostly demographic } \\
\text { characteristics) }\end{array}$ & Data and Country \\
\hline $\begin{array}{l}\text { Mazzanti and Montini } \\
\text { (2006) }\end{array}$ & Per-capita water consumption & $\begin{array}{ll}\text { - } & \text { price (central price of the tariff structure, medium block) }(-) \\
\text { - } & \text { income }(+) \\
\text { - } & \text { population } \\
\text { - } & \text { population density } \\
\text { - } & \text { household size } \\
\text { - } & \text { percentage of population above } 65 \text { years } \\
\text { - } & \text { percentage of population below } 19 \text { years } \\
\text { - } & \text { altitude (interaction term with population) }(-) \\
\text { - } & \text { elderly ratio }\end{array}$ & $\begin{array}{l}\text { Emilia-Romagna, Italy } \\
\text { (1998 to 2001) }\end{array}$ \\
\hline $\begin{array}{l}\text { Nauges and Thomas } \\
(2000)\end{array}$ & $\begin{array}{l}\text { water consumption in local } \\
\text { community }\end{array}$ & $\begin{array}{ll}\text { - } & \text { marginal price }(-) \\
\text { - } & \text { average price }(-) \\
\text { - } & \text { income }(+) \\
\text { - } & \text { rainfall during summer } \\
\text { - } & \text { population density } \\
\text { - } & \text { proportion of inhabitants aged more than } 60 \text { years }(-) \\
\text { - } & \text { proportion of households consisting of one or two members } \\
\text { - } & \text { local economic activity }(+) \\
\text { - } & \text { proportion of single-unit houses }(-) \\
\text { - } & \text { proportions of homes with a bath (significant when average price model used) } \\
\text { - } & \text { proportion of households with one or more cars } \\
\text { - } & \text { proportion of housings built before } 1949 \text { (insignificant when average price } \\
\text { - } & \text { podel used) }(+) \\
\end{array}$ & $\begin{array}{l}\text { panel data from } 116 \text { communities, } \\
\text { eastern France } \\
\text { (1988 to 1993) }\end{array}$ \\
\hline $\begin{array}{l}\text { Nauges and Thomas } \\
\text { (2003) }\end{array}$ & $\begin{array}{l}\text { annual consumption of water } \\
\text { (intertemporal choice) }\end{array}$ & $\begin{array}{ll}\text { - } & \text { lagged consumption }(+) \\
\text { - } & \text { price of water }(-) \\
\text { - } & \text { income }(+)\end{array}$ & $\begin{array}{l}\text { panel data from } 116 \text { communities, } \\
\text { eastern France } \\
\text { (1988 to 1993) }\end{array}$ \\
\hline
\end{tabular}




\begin{tabular}{|c|c|c|c|}
\hline Authors (year) & Dependent variables & $\begin{array}{c}\text { Independent variables other than policy variables (mostly demographic } \\
\text { characteristics) }\end{array}$ & Data and Country \\
\hline $\begin{array}{l}\text { Nieswiadomy } \quad \text { and } \\
\text { Molina (1989) }\end{array}$ & monthly water consumption & $\begin{array}{ll}\text { - } & \text { price }(-) \\
\text { - } & \text { difference variable }(+) \\
\text { - } & \text { income }(+) \\
\text { - } & \text { lawn size }(+) \\
\text { - } & \text { weather }(+) \\
\text { - } & \text { house size }(+)\end{array}$ & $\begin{array}{l}\text { monthly data, Denton, Texas } \\
\text { (1976 to 1985) }\end{array}$ \\
\hline Pint (1999) & bi-monthly water use & $\begin{array}{ll}\text { - } & \text { marginal price }(-) \\
\text { - } & \text { difference variable }(+) \\
\text { - } & \text { house size }(+) \\
\text { - } & \text { lot size }(+) \\
\text { - } & \text { precipitation }(-) \\
\text { - } & \text { lagged precipitation }(-) \\
\text { - } & \text { monthly average temperature }(+) \\
\text { - } & \text { lagged average temperature }(+)\end{array}$ & $\begin{array}{l}\text { Alameda County Water District, } \\
\text { California } \\
\text { (Jan } 1982 \text { to Jul 1992) }\end{array}$ \\
\hline
\end{tabular}




\begin{tabular}{|c|c|c|c|}
\hline Authors (year) & Dependent variables & $\begin{array}{l}\text { Independent variables other than policy variables (mostly demographic } \\
\text { characteristics) }\end{array}$ & Data and Country \\
\hline $\begin{array}{ll}\text { Renwick } & \text { and } \\
\text { Archibald (1998) }\end{array}$ & $\begin{array}{l}\text { water consumption per } \\
\text { household }\end{array}$ & $\begin{array}{ll}\text { - } & \text { lagged marginal price }(-) \\
\text { - } & \text { income }(+) \\
\text { - } & \text { lagged difference variable }(+) \\
\text { - } & \text { dummy for Santa Barbara's landscape irrigation use restriction policy (-) } \\
\text { - } & \text { dummy for Goleta's quantity-related water allocation policy }(-) \\
\text { - } & \text { dummy for low-density households }(+) \\
\text { - } & \text { dummy for medium-density households }(-) \\
\text { - } & \text { number of members in a household }(+) \\
\text { - } & \text { number of faucets in the household }(+) \\
\text { - } & \text { cumulative monthly rainfall } \\
\text { - } & \text { adoption of traditional irrigation technologies by the household }(+) \\
\text { - } & \text { adoption of water-efficient irrigation technologies by the household }(-) \\
\text { - } & \text { adoption of low-flow showerheads by the households (-) } \\
\text { - } & \text { adoption of low-flow toilets by the households }(-) \\
\text { - } & \text { consumer price index }(-)\end{array}$ & $\begin{array}{l}\text { household survey data, } \\
\text { Santa Barbara and Goleta, } \\
\text { California } \\
\text { (1985 to 1992) }\end{array}$ \\
\hline
\end{tabular}




\begin{tabular}{|c|c|c|c|}
\hline Authors (year) & Dependent variables & $\begin{array}{c}\text { Independent variables other than policy variables (mostly demographic } \\
\text { characteristics) }\end{array}$ & Data and Country \\
\hline Renwick et al. (1998) & $\begin{array}{l}\text { average household monthly } \\
\text { water use }\end{array}$ & $\begin{array}{ll}\text { - } & \text { marginal price (-) } \\
\text { - } & \text { difference variable } \\
\text { - } & \text { public information campaigns dummy (-) } \\
\text { - } & \text { subsidies dummy for adopting water-efficient technologies } \\
\text { - } & \text { free retrofit kit distribution dummy }(-) \\
\text { - } & \text { rationing of water dummy(-) } \\
\text { - } & \text { restriction on some types of water use dummy (-) } \\
\text { - } & \text { compliance dummy for certain conservation measures } \\
\text { - } & \text { limited irrigation dummy }(-) \\
\text { - } & \text { irrigation dummy }(+) \\
\text { - } & \text { average monthly gross household income }(+) \\
\text { - } & \text { average household lot size }(+) \\
\text { - } & \text { average maximum air temperature }(+) \\
\text { - } & \text { precipitation }\end{array}$ & $\begin{array}{l}\text { eight urban water agencies, } \\
\text { California, USA } \\
\text { (1989 to 1996) }\end{array}$ \\
\hline $\begin{array}{l}\text { Schefter and David } \\
\text { (1985) }\end{array}$ & $\begin{array}{l}\text { total quantity of water } \\
\text { delivered to residential users }\end{array}$ & $\begin{array}{ll}- & \text { marginal price }(-) \\
- & \text { income }(+) \\
\text { - } & \text { difference variable }\end{array}$ & $\begin{array}{l}\text { aggregate data, } \\
131 \text { Wisconsin communities }\end{array}$ \\
\hline
\end{tabular}




\begin{tabular}{|c|c|c|c|}
\hline Authors (year) & Dependent variables & $\begin{array}{c}\text { Independent variables other than policy variables (mostly demographic } \\
\text { characteristics) }\end{array}$ & Data and Country \\
\hline $\begin{array}{l}\text { Strand and Walker } \\
(2005)\end{array}$ & average water consumption & $\begin{array}{ll}\text { - } & \text { access to metered tap water: } \\
\text { - } & \text { marginal water price } \\
\text { - } & \text { average price of water }(-) \\
\text { - } & \text { household income }(+) \\
\text { - } & \text { children }(+) \\
\text { - } & \text { adults }(+) \\
\text { - } & \text { with telephone }(+) \\
\text { - } & \text { value of house }(+) \\
& \text { dummies for service hours (rationing of water through reduced service } \\
\text { no access to tap water: } \\
\text { - } & \text { water price }(-) \\
\text { - } & \text { truck water time hauling cost }(-) \\
\text { - } & \text { other water time hauling cost }(-) \\
\text { - } & \text { household size }(+) \\
\text { - } & \text { household income }(+)\end{array}$ & $\begin{array}{l}\text { survey data, } \\
17 \text { Central American cities }\end{array}$ \\
\hline Taylor et al. (2004) & $\begin{array}{l}\text { quantity of domestic water } \\
\text { demanded }\end{array}$ & $\begin{array}{ll}\text { - } & \text { price (marginal price, average revenue) }(-) \\
\text { - } & \text { highest annual temperature }(+) \\
\text { - } & \text { monthly precipitation } \\
\text { - } & \text { income }(+) \\
\text { - } & \text { water conservation program dummy } \\
\text { - } & \text { various pricing dummy [increasing block }(-), \text { decreasing block }(+), \text { un- } \\
& \text { metered (+) compared to constant rate] }\end{array}$ & $\begin{array}{l}\text { cross-sectional data, } \\
34 \text { utilities from Colorado water } \\
\text { districts }\end{array}$ \\
\hline
\end{tabular}




\begin{tabular}{|c|c|c|c|}
\hline Authors (year) & Dependent variables & $\begin{array}{c}\text { Independent variables other than policy variables (mostly demographic } \\
\text { characteristics) }\end{array}$ & Data and Country \\
\hline $\begin{array}{l}\text { Whittington et al. } \\
\text { (1990) }\end{array}$ & $\begin{array}{l}\text { probability that a household's } \\
\text { WTP for a public stand-post } \\
\text { (private connection) falls } \\
\text { within a specific interval }\end{array}$ & $\begin{array}{ll}\text { - } & \text { household wealth index }(+) \\
\text { - } & \text { dummy for household foreign income } \\
\text { - } & \text { dummy for occupation index (farmer or non-farmer) (significant for private } \\
\text { - } & \text { connections) } \\
\text { - } & \text { distansehold education level }(+) \\
\text { - } & \text { quality index of existing source }(+) \\
\text { - } & \text { dummy for sex (male }=1 \text { ) (insignificant for private connections) }(-)\end{array}$ & $\begin{array}{l}\text { Laurent, southern Haiti } \\
\text { (1986) }\end{array}$ \\
\hline \multirow[t]{2}{*}{ Wong (1972) } & \multirow[t]{2}{*}{$\begin{array}{lll}\text { average } & \text { per-capita } & \text { water } \\
\text { demand } & & \end{array}$} & $\begin{array}{ll}\text { - } & \text { price of water (insignificant for Chicago) }(-) \\
\text { - } & \text { average household income (insignificant for suburbs) }(+) \\
\text { - } & \text { average summer temperature }(+)\end{array}$ & $\begin{array}{l}\text { time series analysis, Chicago and } \\
\text { nearby communities, Illinois } \\
\text { (1951 to 1961) }\end{array}$ \\
\hline & & $\begin{array}{ll}\text { - } & \text { price of water (insignificant for smallest community) (-) } \\
\text { - } & \text { average household income (insignificant for smaller communities) }(+)\end{array}$ & $\begin{array}{l}\text { cross-sectional analysis with four } \\
\text { community sizes }\end{array}$ \\
\hline Woo (1994) & monthly per-capita water use & $\begin{array}{ll}\text { - } & \text { monthly average rate of water }(-) \\
\text { - } & \text { monthly per-capita income }(+) \\
\text { - } & \text { monthly supply hours of water (during the supply interruptions) }(+) \\
\text { - } & \text { rainfall - evaporation } \\
\text { - } & \text { average temperatures }(+) \\
\text { - } & \text { quarter dummy }(\mathrm{Q} 1)(-) \\
\text { - } & \text { quarter dummy }(\mathrm{Q} 2)(+) \\
\text { - } & \text { quarter dummy }(\mathrm{Q} 3)(+) \\
\text { - } & \text { number of calendar days per month }(+)\end{array}$ & $\begin{array}{l}\text { Hong Kong } \\
\text { (1973 to 1990) }\end{array}$ \\
\hline
\end{tabular}


TABLE C.3: Summary of income and price elasticities of demand for residential water

\begin{tabular}{|c|c|c|c|c|}
\hline Authors (year) & Policy program & Price elasticity & Income elasticity & Comments \\
\hline $\begin{array}{l}\text { Billings and Agthe } \\
\text { (1980) }\end{array}$ & - $\quad$ increasing block rates & $\begin{array}{l}-0.267 \quad \text { (double-log } \\
\text { model) } \\
-0.49 \text { (linear model) }\end{array}$ & & marginal price used \\
\hline $\begin{array}{lr}\text { Chicoine } & \text { and } \\
\text { Ramamurthy (1986) }\end{array}$ & - $\quad$ declining block price schedule & $\begin{array}{l}-0.47 \text { (for marginal } \\
\text { price) }\end{array}$ & & \\
\hline Dandy et al. (1997) & $\begin{array}{l}\text { two-part tariff (free allowance with a } \\
\text { fixed charge }+ \text { unit price of water beyond } \\
\text { the allowance level) }\end{array}$ & $\begin{array}{l}-0.28 \text { (short-run) } \\
-0.63 \text { to }-0.77 \text { (long- } \\
\text { run) }\end{array}$ & $\begin{array}{l}0.14 \text { (short-run) } \\
0.32 \text { to } 0.38 \text { (long-run) } \\
\text { (property value used as a } \\
\text { proxy for income) }\end{array}$ & $\begin{array}{l}\text { price elasticities are above free allowance level; under free } \\
\text { allowance level, elasticity is zero as marginal price is zero. }\end{array}$ \\
\hline Espiñeira (2000) & - $\quad$ increasing block rate & -0.12 to -0.16 & & various model specifications used \\
\hline $\begin{array}{ll}\text { Espiñeira } & \text { and } \\
\text { Nauges (2004) }\end{array}$ & $\begin{array}{ll}- & \text { increasing block rate } \\
\text { - } & \text { restricted daily water supply hours during } \\
& \text { severe drought periods } \\
\text { - } & \text { temporary outdoor use bans }\end{array}$ & -0.10 & 0.10 & on average \\
\hline Gaudin (2006) & $\begin{array}{l}\text { - using clear pricing information on water } \\
\text { bills }\end{array}$ & $\begin{array}{l}-0.37 \\
-0.51 \quad \text { (with price } \\
\text { information on water } \\
\text { bill) }\end{array}$ & 0.30 & \\
\hline Headley (1963) & & & $\begin{array}{l}\text { cross-sectional: } \\
1.49 \text { (in 1950) } \\
1.24 \text { (in 1959) } \\
\text { time-series: } \\
\begin{array}{l}0.00136 \text { to } 0.4035 \text { (for } 14 \\
\text { cities) }\end{array}\end{array}$ & water use regressed on consumers' income \\
\hline $\begin{array}{l}\text { Mazzanti and } \\
\text { Montini (2006) }\end{array}$ & - $\quad$ increasing block-rate structure & -0.99 to -1.33 & 0.40 to 0.71 & $\begin{array}{l}\text { introduction of time effects or other socio-economic } \\
\text { variables reduces significance level of income variable, } \\
\text { and sometimes makes it insignificant. }\end{array}$ \\
\hline
\end{tabular}




\begin{tabular}{|c|c|c|c|c|}
\hline Authors (year) & Policy program & Price elasticity & Income elasticity & Comments \\
\hline $\begin{array}{l}\text { Nauges and Thomas } \\
(2000)\end{array}$ & $\begin{array}{ll} & \text { two-part tariff } \\
\text { - } & \text { existence of own water meter in single- } \\
& \text { unit houses }\end{array}$ & -0.22 & 0.1 & \\
\hline $\begin{array}{l}\text { Nauges and Thomas } \\
\text { (2003) }\end{array}$ & - $\quad$ two-part tariff & $\begin{array}{l}-0.26 \text { (short-run) } \\
-0.40 \text { (long-run) }\end{array}$ & 0.51 (long-run) & \\
\hline $\begin{array}{l}\text { Nieswiadomy } \\
\text { Molina (1989) }\end{array}$ & $\begin{array}{ll}- & \text { increasing block rate (1976 to } 1980) \\
\text { - } & \text { decreasing block rate (1981 to } 1985 \text { ) }\end{array}$ & -0.36 to -0.86 & & 2SLS and IV techniques used \\
\hline Pint (1999) & - $\quad$ increasing block rate & $\begin{array}{l}\text { heterogeneous } \\
\text { preferences model: } \\
-0.04 \text { to }-0.14 \text { (summer) } \\
-0.07 \text { to }-0.29 \text { (winter) } \\
\text { two-error model: } \\
-0.20 \text { to }-0.47 \text { (summer) } \\
-0.33 \text { to }-1.24 \text { (winter) }\end{array}$ & & $\begin{array}{l}\text { less elastic demand curve with heterogeneous- preferences } \\
\text { model than with two-error model }\end{array}$ \\
\hline
\end{tabular}




\begin{tabular}{|c|c|c|c|c|}
\hline Authors (year) & Policy program & Price elasticity & Income elasticity & Comments \\
\hline $\begin{array}{l}\text { Renwick and } \\
\text { Archibald (1998) }\end{array}$ & $\begin{array}{l}\text { - } \\
\text { - } \quad \text { dummieasing block-rate pricing } \\
\text { irrigation use restriction policy } \\
\text { - dummy for Goleta's quantity-related } \\
\text { water allocation policy } \\
\text { - dummy for the low-flow toilet and } \\
\text { showerhead subsidy programs } \\
\text { - adoption of traditional irrigation } \\
\text { technologies by the household } \\
\text { - adoption of water-efficient irrigation } \\
\text { technologies by the household } \\
\text { - adoption of low-flow showerheads by the } \\
\text { households } \\
\text { - adoption of low-flow toilets by the } \\
\text { households }\end{array}$ & $\begin{array}{l}-0.33 \text { (short-run) } \\
-0.39 \text { (long-run) }\end{array}$ & 0.36 & 2SLS estimation methods used \\
\hline $\begin{array}{l}\text { Renwick et } \quad \text { al. } \\
\text { (1998) }\end{array}$ & $\begin{array}{ll}\text { - } & \text { uniform and block-rate pricing } \\
\text { - } & \text { public information campaigns } \\
\text { - } & \text { subsidies for adopting water-efficient } \\
& \text { technologies } \\
\text { - } & \text { free retrofit kit distribution } \\
\text { - } & \text { rationing of water } \\
\text { - } & \text { restriction on some types of water use } \\
\text { - } & \text { compliance for certain conservation } \\
& \text { measures }\end{array}$ & $\begin{array}{l}-0.16 \\
-0.20 \text { (June to August) }\end{array}$ & 0.25 & \\
\hline $\begin{array}{l}\text { Strand and Walker } \\
(2005)\end{array}$ & 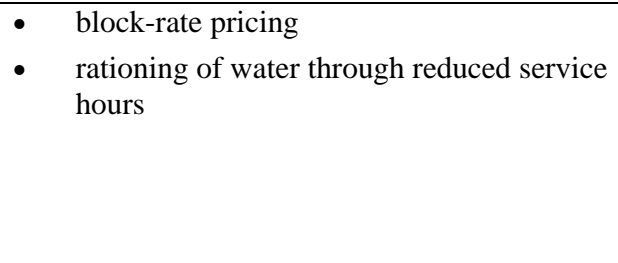 & $\begin{array}{l}\text { With tap water access: } \\
-0.1 \text { to }-0.2 \text { (using MP) } \\
-0.3 \text { (using both MP } \\
\text { and AP) } \\
\text { No tap access: } \\
-0.10\end{array}$ & $\begin{array}{l}<0.1 \\
\text { (same for both groups of } \\
\text { households) }\end{array}$ & \\
\hline
\end{tabular}




\begin{tabular}{|c|c|c|c|c|}
\hline Authors (year) & Policy program & Price elasticity & Income elasticity & Comments \\
\hline Taylor et al. (2004) & $\begin{array}{l}\text { - } \quad \text { various price schedules (flat- rate pricing, } \\
\text { increasing block, decreasing block, and } \\
\text { un-metered fixed fees) } \\
\text { - } \quad \text { water conservation programs }\end{array}$ & $\begin{array}{l}-0.297 \text { (with MP) } \\
-0.403 \text { (with AP) }\end{array}$ & 0.38 & TSLS estimation process \\
\hline \multirow[t]{2}{*}{ Wong (1972) } & $\begin{array}{ll}- & \text { flat rate of water per } 1000 \text { gallons } \\
\text { (Chicago) } \\
\text { - } & \text { block rates (suburban communities) }\end{array}$ & -0.02 to -0.28 & 0.20 to 0.26 & time series \\
\hline & & -0.26 to -0.82 & 0.48 to 1.03 & cross-sectional \\
\hline Woo (1994) & $\begin{array}{ll}\text { - } & \text { restricted supply of water (service } \\
\text { interruptions) } \\
\text { - } & \text { inverted block-rate pricing }\end{array}$ & -0.4684 & 0.2354 & based on double-log/AR(1) specification \\
\hline
\end{tabular}

Note: marginal prices (MP), average prices (AP). 
